\title{
Cancer Research.*
}

$\mathrm{T}$ HE British Empire Cancer Campaign continues to subsidise a great variety of investigations into the cause and treatment of cancer, and the progress which is being made is summarised fairly fully in the recent annual report.

The greatest practical advance in the cancer problem which has been made in recent years is the cumulative demonstration that tar and soot and, generally, the products of burned coal, together with certain mineral oils, have an exceptional efficiency in causing malignant tumours when they are applied to the tissues of men and animals over long periods of time. The evidence comes partly from experiments on animals and partly from studying the relation of cancer to occupation and mode of life. The two lines of inquiry are entirely concordant in implicating burnt coal, and they provide sanitarians with a clear indication for preventing some forms of cancer.

For experimental purposes tar has generally been used as a carcinogenic agent, and a good deal of research has gone into the obvious question as to what particular substance in that complicated mixture is responsible. Most of the components have been tried and found to be inert, and no pure substance has been found which had anything like the effect of tar. It seems now, however, that Mr. I. Hieger and Dr. J. W. Cook, at the Fulham Cancer Hospital, have made substantial progress by following up the observation that benzanthracene and cancer-producing tar have similar fluorescent spectra. They have since found that $1: 2: 5: 6$ dibenzanthracene in a high state of chemical purity readily produces cancer when applied in quite small concentrations to the skin of mice, and they have thus provided a most valuable method for the analysis of the precise mechanism by which such cancers are produced. Whether this or some analogous compound is in fact the active agent in tars and mineral oils is not known.

There is at any rate no obvious justification for the statement made in the Report of the Grand Council (though not, we notice, by the workers themselves) that " the essential molecular structure of the cancer-producing agent in tar and other cancer-producing substances " has been determined. It may well be that the agent is not the same in all tars and carcinogenic oils. Cancer of the skin may

* British Empire Cancer Campaign, Eighth Annual Report of the Grand Council, presented at the meeting held at the House of Lords on July 20. (London: British Empire Cancer Campaign.) be produced experimentally by X-rays, freezing, burning, acids, and other procedures which irritate the skin and have no 'substance' in common, though they may be similarly active because they all lead to the tissues producing the same active agent.

Another inquiry which relates the chemical constitution of a substance to its capacity to cause the growth of cells has been carried out by Dr. J. S. Young at Leeds. Solutions of simple salts injected into the pleural sac stimulate the cells lining the marginal alveoli of the lung to proliferate, and their efficiency in a general way varies with the valency of the metal: aluminium is more effective than calcium, and calcium than sodium. To these interesting facts he has now added the observation that cells which have reacted once are refractory. to a further application of the stimulating salt for about three weeks. This resistance may also be induced by a series of injections of gradually increasing strength such that no reaction is at any time produced: ultimately the cells tolerate a concentration of the salt twice as great as that required to cause a reaction in a normal animal. This kind of cellular immunity to irritating substances is little understood and needs further investigation, possibly on simpler material than that used by Dr. Young.

While these and other data indicate quite clearly that cancer is essentially a local disease due to local causes, it is also evident that general or constitutional factors may be involved. In the present report, for example, it is shown that some strains of mice respond more quickly to the application of tar than others, and mention is made of a breed in which tumours appear 'spontaneously' in an extraordinarily large percentage of animals. Dr. C. E. Dukes also presents the pedigrees of a number of human families in which a hereditary predisposition to the development of multiple innocent tumours of the bowel is plainly shown: in many cases these growths form the starting point of cancers which appear at an exceptionally early age. It may probably be presumed that by deliberate dysgenic mating, strains of men might be produced with a special tendency to respond to external irritants by the growth of cancers, but there is no evidence that the constitutional factor is of any substantial importance in an ordinary human society, except in a few special instances such as that studied by Dr. Dukes.

\section{Obituary.}

\section{Dr. Joan B. Procter.}

GELDOM has the triumph of force of mind over $\$$ physical weakness been more vividly illustrated than in the case of Dr. Joan Beauchamp Procter, curator of reptiles and amphibians in the Gardens of the Zoological Society of London, who died at her residence in Regent's Park on Sept. 20, at the age of thirty-four years.
Fragile from birth, she still persisted in her duties, first at the Natural History Museum and later at the Zoo, when the majority in like position would excusably have retired.

From quite early days she had dedicated her abilities to the subject which was to become her life's work. As a child she showed an extraordinary interest in reptile and amphibian life,

No. 3233, VoL. 128] 